\title{
Weitere Beiträge zur Kenntnis des Spätglazials
}

\author{
Von Hugo Gross, Bamberg
}

\begin{abstract}
Zusammenfassung: Einige während und nach der Drucklegung meines Aufsatzes über das Alleröd-Interstadial als Leithorizont der letzten Vereisung (1954) veröffentlichte Arbeiten und sonst bekannt gewordene Befunde werden zur Vervollständigung meines Aufsatzes auf den Stand von Ende 1954 ausgewertet und einige Berichtigungen vorgenommen, insbesondere für die Karte und die Übersichtstabelle.
\end{abstract}

S u m ma ry : Some papers published during and after the printing of my treatise of 1954 concerning the Alleröd interstadial and some statements otherwise made known are evaluated in order to supplement my above paper, especially its map and table, by the close of the year 1954.

1. Das Alleröd-Interstadial: Neue Vorkommen sind: in Dänemark Vallensgaard Mose auf Bornholm (Johs. Iversen 1954 Pl. XI), in Belgien Vivier Fagnoul (Fagne Wallone $\mathrm{O}$ von Botrange) nach $\mathrm{F}$. FloRschütz und E. van Oye mit geringen Pollenwerten von Alnus, Carpinus, Quercus, Tilia, Ulmus und Corylus (wohl durch den Bohrer verschleppt) und höheren von Pinus, Betula und Salix (J. Sauvage 1954); in Deutschland: bei Elmshorn NW von Hamburg (R. Hallik \& E. Grube 1954); Brothener Ufer bei Travemünde (P. RANGE 1951). In O'sterreich: Egelseegebiet (SalzachVorlandgletscher) NO der Stadt Salzburg (Eva von Lürzer 1954. Alleröd mit LaacherSee-Tuff im Kirchhain-Amöneburger Becken im Raume Marburg-Gießen an 1 Fundstelle bei Kirchhain und an 2 Fundstellen bei Plausdorf (H. D. LANG 1954); meine Angabe (Abb. 1 und S. 192) „Moor im Grunewald bei Berlin“ ist zu streichen, da sie auf einer ungenauen Information beruht, es soll sich um Staubschichten, die nichts mit Laach zu tun haben, in wärmezeit $\mathrm{lich}$ e Moorschichten handeln.

2. Das Bölling-Interstadia 1: Meine Angabe (1954 S. 195), daß dieses Interstadial den dänischen Fachleuten noch problematisch sei, beruht auf einem Mißverständnis meines Gewährsmannes. Herr Dr. Johs. Iversen teilte mir (durch Brief vom 3. 12. 54) mit, daß er auf der vegetationsgeschichtlichen Konferenz in Kopenhagen 1953 „zwei neue, gründlichere Pollendiagramme aus anderen Profilen vom Böllingsee demonstrierte, wodurch die Bölling-Oszillation klar bewiesen wird, und es wurden keinerlei Bedenken in der Diskussion vorgebracht (vergl. Johs. Ivensen 1954 S. 94 und Pl. X und I. Brandt 1954). Wie mir Herr Dr. Iversen weiter mitteilte, hat „K. FAeGrr später von seinem Bröndmyr-Interstadial Abstand genommen. Neue Untersuchungen sollten aber in Jaeren ausgeführt werden“. Undeutlich ist das Bölling-Interstadial in einem Pollendiagramm bei Elmshorn im Altmoränengebiet NW von Hamburg registriert (R. HAllik \& E. Grube 1954). In einem Spätglazialdiagramm aus dem Egelseegebiet NO der Stadt Salzburg (Eva von Lürzer 1954) ist nach sehr langer waldloser Phase eine geringe Wärmeschwankung Ib (mit erster Birkenausbreitung, erstem Fichtenvorstoß und vorübergehender NBP-Abnahme) registriert, die nach Ansicht der Verfasserin nichts mit dem Ib des Nordens zu tun hat; sie dürfte aber doch der BöllingSchwankung entsprechen.

Von der Fundstelle Poggenwisch bei Ahrensburg i. Holst, mit Artefakten der Hamburger Kultur Stufe II ist von R. SсHÜтRUMPF (1954) das Pollendiagramm zur 6. Hauptversammlung der Deutschen Quartärvereinigung in Segeberg vom 20.-25. 9. 54 fertiggestellt worden; nach diesem Pollendiagramm ist das Bölling-Interstadial etwas jünger, als unsprünglich angegeben war, nämlich etwas jünger als die Fundschicht der Hamburger Stufe II, für die in Washington mit einer verbesserten C 14-Methode (mit Verwendung von Azetylen statt des festen Kohlenstoffs) die Zeitstellung $15150 \pm 350$ 
Jahre vor heute $=$ ca. $13200 \pm 350$ v. Chr. ermittelt worden ist (A. Ruśt 1954); damit ist also ein Terminus post quem für das Bölling-Interstadial festgestellt, der nach H. Schmitz beträchtlich jünger als die Hauptmoräne des Pommerschen Stadiums ist (H. Gross 1954 S. 194, 195), so daß das Bölling-Interstadial nicht in eine W II/IIISchwankung fallen kann.

3. Die Alteste Dryaszeit: Die Jüngere Hamburger Kultur Stufe II von der Poggenwisch bei Ahrensburg gehört also nach R. SchütrumpF (1954) wie die Ältere Hamburger Kultur (I) in den waldfreien Abschnitt I SchütrumpF = Ia Iversen des Spätglazials; „die dazu gehörigen Pollenspektren unterscheiden sich jedoch von denen aus der Alteren Hamburger Stufe durch bereits niedrigere Nichtbaumpollenprozente, was auf jüngeres Alter hinweist". Für die Altere Hamburger Kultur (I) ist inzwischen (nach briefl. Mitteil. von Herrn Dr. A. Rust vom 22. 11. 54) in Washington durch die C 14-Bestimmung die Zeitstellung $15800 \pm 800$ Jahre vor heute $=$ ca. $13800 \pm 800$ v. Chr. ermittelt worden, sie gehört also weder in ein W I/II-, noch in ein W II/IIIInterstadial, denn die Alteste Dryaszeit ist nach den Befunden von H. Schmitz bei Heiligenhafen und Travemünde beträchtlich jünger als die Hauptmoräne des Pommerschen Stadiums (H. Gross 1954 S. 195). Der Eisrückzug von einer etwas jüngeren Moräne als Moräne M ist von E H. DE GEen $(1951,1954)$ auf Grund einer Warwenmessung (in der Ziegelei Bunte Kuh bei Lübeck) und -konnektierung mit S-Schonen mit 15730 Jahren vor heute $=$ ca. 13800 v. Chr. datiert; zwischen dem Beginn der Altesten Dryaszeit und dem Beginn des Eisrückzugs von der Hauptmoräne M liegen noch jüngere Endmoränen des Pommerschen Stadiums und in diesen Interstadialbildungen, es muß also der Eisrückzug von der Hauptmoräne $M$ er he blich vor dem Meiendorfer Datum (15800 \pm 800 vor heute) erfolgt sein.

Das von H. Schmitz bei Heiligenhafen pollenanalytisch untersuchte Profil, das mit der Altesten Dryaszeit ( 2 oder 3 Proben) beginnt, ist am Ansatzpunkt der Nehrung Steinwarder unmittelbar hinter dem Strandwall abgebohrt; etwa 700-800 m westlich und südlich davon stehen Endmoränenzüge an, die nach K. GrIPP Stauchendmoränen sind; es liegen keine Anzeichen dafür vor, daß die untersuchten Beckenabsätze durch Vorschüttungssande oder Fließerden bei der Bildung der jüngeren Endmoränen berührt wurden (H. Schmitz, briefl. Mitteil. vom 26. 3. 54). Die von H. Schmitz bei Heiligenhafen untersuchte spätglaziale Schichtenfolge ist nach den geomorphologischen Übersichtskarten von G. SEIFERT (1954) jünger als der E-W-Eisvorstoß der Eisabbauphase III, der dort und auf Fehmarn den „oberen“ Geschiebemergel hinterließ; seine Richtung hat G. SEIFERT durch die Untersuchung des mikroskopischen Geschiebemergelkorngefüges ermittelt. Für die älteste Eisabbau-Phase I hat G. SEIFERT einen N-S- bzw. NESW-Vorstoß, für die Phase II einen gehemmten N-S-Vorstoß und einen lokalen W-EVorstoß festgestellt. Es liegt also die Vermutung nahe, daß die jüngste Phase III der gotiglaziale Eisvorstoß im Sinne von G. und E. H. DE GeER ist, dessen Beginn G. DE GEER mit ca. 13200 v. Chr. = 15150 Jahre vor heute datiert hat; ist diese Vermutung richtig, so müßte der gotiglaziale Eisvorstoß noch vor $15800 \pm 800$ Jahren vor heute $=$ ca. $13800 \pm 800$ v. Chr. = Zeitstellung der Meiendorfer Kulturschicht der Altesten Dryaszeit begonnen haben, wenn bei Heiligenhafen die Alteste Dryaszeit von derselben Länge wie in Meiendorf erfaßt ist.

Nun hat G. H. BRüCKNER (1954) Artefakte der Jüngeren Hamburger Kultur (II) wie bei der Fundstelle Poggenwisch bei Ahrensburg i. Holst., also $15150 \pm 350$ Jahre alt, nicht abgerollt in der Küstensteilwand beim Ostseebad Grömitz (am NO-Strand der Lübecker Bucht $S$ von Heiligenhafen) in 4-4,80 m Tiefe in der fast senkrechten Grundmoränenwand gefunden; er nimmt daher, den Kieler Geologen, R. SchütrumpF (1954) und A. Rust (1954) folgend, an, daß hier ein Renntierjägerlager der genannten Kultur von einem Inlandeisvorstoß überfahren sei, nach R. SCHütrumpF (1954) in der 
Altesten Dryaszeit zwischen $15150 \pm 350$ Jahren vor heute und dem Bölling-Interstadial. Dieser Eisvorstoß müßte jünger sein als die Eisabbau-Phase III von G. SEIFERT bei Heiligenhafen. Es ist nicht bekannt, daß eine Gletscherzunge der Eisabbau-Phase III oder eine etwas jüngere das Gebiet von Grömitz überfuhr. Es muß daher, wie ich schon 1954 (S. 204) ausgeführt habe, mit der Möglichkeit gerechnet werden, daß die Grömitzer Artefakte durch eine Schichtenstörung im Zusammenhang mit Tieftauen von verschüttetem Toteis sekundär in die Grundmoräne geraten sind; derartige Rutschungen sind ja im Spätglazial außerordentlich häufig gewesen. Es ist daher unbedingt notwendig, durch die Untersuchung des makro- und mikroskopischen Geschiebemergelkorngefüges des Grömitzer Grundmoränenprofils festzustellen, ob es wirklich ungestört ist. Auch in diesem Falle wäre der Fund von Grömitz außerordentlich wichtig, da er erstmals einen Inlandeisvorstoß im absoluten Zeitmaß zu datieren ermöglichen würde.

Zeitlich entspricht der Hamburger Kultur NW-Deutschlands aller Wahrscheinlichkeit nach die Kulturhinterlassenschaft an der Schussenquelle in SW-Deutschland, die K. J. NARR (briefl. Mitteil. 1954) in das Spätmagdalénien (VIa, mittlere Phase) stellt. Die 1866 entdeckte Freilandstation ist in 10 Tagen von O. FraAs (1867) mit „Durchwühlung der Culturschichte“ ausgegraben worden, wobei leider die Funde nicht schichtenweise getrennt wurden. In einer verdienstvollen Arbeit hat K. BERTsCH (1953/54) erneut versucht, die Zeitstellung dieser ersten auf deutschem Boden entdeckten paläolithischen Fundstelle zu ermitteln. Diese lag nach K. Bertsch unter dem Rande des Würmeises zur Zeit seiner größten Ausdehnung und war nach seinem Abrücken von einem Eisstausee bedeckt, der nach $S$ erst abfließen konnte, als der Eisrand nach Eschach S von Ravensburg gerückt war (Stadium W III, zeitlich dem Pommerschen Stadium entsprechend). K. Bertsch nimmt an, daß sogleich nach dem Trockenfallen des Schussenquellengebiets die Renntierjäger-Station entstand, so daß diese in die letzte Stufe der letzten Eiszeit (W III) vor 23000 Jahren gehört.

Gegen diese Datierung sprechen aber geologische und paläontologische Befunde. Erstens mußte zunächst die Schlucht der Schussenquelle im Kies erodiert werden, zweitens mußte am Boden dieser Schlucht durch Tieftauen eines verschütteten Toteisblocks im Untergrund die von steilen Kieswänden umgebene Grube entstehen, die mit den petrographisch so verschiedenen Fundschichten ausgefüllt war und daher auf O. FraAs (1867 S. 58, 59) den Eindruck „einer von Menschenhand gegrabenen Grube“, d. h. einer Abfallgrube oder eines Kehrichthaufens machte. Für die Deutung der Grube als Toteisloch (im voralpinen Vereisungsgebiet für die Moorgeologie anscheinend eine große Seltenheit) sprechen folgende Befunde: an ihrem Rande wurde eine halbbearbeitete Rengeweihstange zerbrochen unter einem 5-6 Zentner schweren erratischen Block gefunden; die ca. 1,20-1,50 m mächtige Kalktuffbank ist über der Grube tief eingedellt; der Torf im Hangenden ist mit Kies von der Wasserscheide teilweise bedeckt (vergl. Tafel II bei O. FraAs 1867; Abb. 2 von K. Bertsch I. c. hat diese Befunde nicht berücksichtigt). Nach den Erfahrungen in Norddeutschland begann das Tieftauen verschütteter Toteisblöcke erst geraume Zeit nach dem Abrücken des Inlandeises von der Hauptmoräne des Pommerschen Stadiums (W III). Drittens handelt es sich um eine Freilandstation wie bei der Hamburger Kultur. Viertens sprechen die faunistischen und botanischen Befunde gegen ein hochglaziales (Schluß von W III) und für ein nicht zu hohes spätglaziales Alter: Elch (K. Bertsch 1. c. S. 23), Braunbär (O. FraAs 1. c. S. 66), Vielfraß und Froschreste (O. FrAAs 1. c. S. 64, 65, 68); von Glazialpflanzen kommen nur Potentilla aurea und Betula nana vor, alle Moosarten kommen noch heute in Oberschwaben vor; ein stricknadelförmiges Holzgerät (O. FraAs 1. c. S. 73) stammt vielleicht von Hippophaë! Eine pollenanalytische Datierung war nicht mög.. lich, da der Blütenstaub in den fast 90 Jahre im Museum trocken aufbewahrten von Kalktuffsand durchsetzten Moosproben infolge dieser Aufbewahrungsart fast vollstän- 
dig sekundär zerstört war. Nur in einem Moostuff und darunterliegendem Ton, die sehr wahrscheinlich der Fundschicht entsprechen, konnte F. FIRBAS (1949 S. 347) ein brauchbares Pollenspektrum ermitteln: Salix 26,2\%, Betula 38,1\%, Pinus 35,7\% (etwa 1. Pinus-Pollenkorn je Präparat $18 \times 18 \mathrm{~mm}$ ), NBP 461,5\% (Gramineen 104\%, Cyperaceen 255\%, Varia 93\%, Farne 2,2\% und Hippophaë oder Helianthemum 9,5\%). Dieses Pollenspektrum zeigt eine waldlose Tundrenzeit an, wahrscheinlich ihren Endabschnitt, wenn die 9,5\% von Hippophaë herrühren; vor allem sprechen Elch, Vielfraß und Braunbär dafür ${ }^{1}$ ). Schließlich spricht die Zunahme der Beimischung von Kalktuffsand über der Tonschicht am Grunde bis zur Bildung einer 1,20-1,50 m mächtigen Kalktuffbank (durch Herauslösung des Kalks aus dem fluvioglazialen Kies der Umgebung) für eine fortschreitende Klimabesserung.

Das Magdalénien VIa der Schussenquelle hat F. Firbas (1949 S. 347) nicht, wie K. Bertsch (1. c. S. 19) irrtümlich angibt, in die Zeit zwischen 12000 und 10000 v. Chr. gestellt, sondern noch in die "Zeit der waldlosen Glazialvegetation" (Diagrammzone Ia) auf Grund des obigen Pollenspektrums. Dieses stimmt gut mit den Pollenspektren von Fundstücken der Alteren Hamburger Kultur I von Meiendorf und Stellmoor (F. FikBaS 1949 S. 347) überein. Die Zeitstellung des Renntierjägerlagers an der Schussenquelle dürfte also kaum nennenswert (sicher nicht 7000 Jahre) vor der des Renntierjägerlagers Meiendorf liegen. Eine Nachprüfung dieser Datierung durch die C 14-Bestimmung von Rengeweihstücken von der Schussenquelle ist dringend erwünscht.

Ist die Zeitstellung des Renntierjägerlagers an der Schussenquelle ca. 16000, die der Station Andernach ca. 12000 Jahre vor heute, so müßte das Spätmagdalénien VIa in Deutschland nicht weniger als ca. 4000 Jahre gedauert haben! Das steht im Widerspruch zu einigen C 14-Daten aus Frankreich (H. Gross 1952, S. 82): eine Kulturschicht aus dem oberen Périgordien oder (weniger wahrscheinlich) sehr altem Magdalénien in Lascaux $15848 \pm 1200$ Jahre vor heute, also ebenso alt wie das Magdalénien VIa von der Schussenquelle! Entweder ist ein Fehler bei der C14-Datierung der Kulturschicht von Lascaux in Chicago oder (weniger wahrscheinlich) der Kulturschichten von der Poggenwisch und Meiendorf (beide in Kalkgyttja) in Washington gemacht worden, es sei denn, daß die datierte Kulturschicht von Lascaux von einer rückständigen Gruppe aus der Zeit des Hochmagdalénien stammt. Auch die drei C 14-Datierungen einer und derselben Magdalénien-Kulturschicht ohne Angabe der Stufe (France I-III) sind sehr widerspruchsvoll (11110 \pm 480 bis $15848 \pm 1200$ Jahre vor heute). Es sind also weitere Datierungen ungestörter französischer Magdalénien-Schichten mit verbesserten C 14-Methoden dringend erforderlich.

4. Das sogen. "Masurische Interstadial “. Mitten in späteiszeitlichen Sandern, die z. T. später zu Endmoränen aufgestaucht wurden, finden sich, wie E. W. Guenther (1954) festgestellt hat, mitunter Ablagerungen von Stillwasser mit spärlicher Molluskenfauna; im Zusammenhang mit solchen Schichten wurde bei Preetz in Holstein das Schädelfragment eines Elchs und bei Schlutup in der Nähe von Lübeck (von mir

1) Wie mir Herr Dr. G. LANG-Karlsruhe brieflich (1. und 5. 3. 55) mitgeteilt hat, konnte Herr Studienrat WaLL-Buchau 1953 durch neue Grabungen einen Rest der von O. FraAs 1866 untersuchten Schichtenfolge an der Schussenquelle auffinden; nach der vorläufigen pollenanalytischen Untersuchung gehört die Hauptfundschicht unter der Kalktuffbank tatsächlich, wie schon F. Firbas festgesellt hat, der Altesten Dryaszeit Ia an, aber ohne Hippophaë, sondern mit Helianthemum cf. alpestre, dessen Pollen 1935 noch nicht vom Hippophaë-Pollen unterschieden wurde; Hippophaë tritt erst über der Kalktuffbank am Grunde der oberen Braunmoostorfschicht auf, in der Fruchtschuppen von Baumbirken vorkommen und Baumpollen in den Pollenspektren überwiegt (Ib). Ausreichendes Material für die $\mathrm{C}^{14}$-Datierung wird zur Verfügung gestellt. 
1954 irrtümlich für Preetz angegeben) ein Riesenhirsch gefunden, wie schon vor etwa 50 Jahren von P. Friedrich. Beide Tiere sind keine ausgesprochenen arktischen Formen und sprechen wie die genannten Süßwasserbildungen für eine vorübergehende Klimabesserung, also ein Interstadial. Diese Funde sind nach E. W. Guenther (1954) jünger als die Hauptmoräne $M$ im Sinne von $K$. GRIPP und älter als die letzten Eisvorstöße, die Schleswig-Holstein erreichten. In Ostholstein hat P. RANGE (1951) interstadiale pflanzenführende Süßwasserbildungen in Vorschïttsanden der Schlutuper Endmoräne und unter Sanddecke gestaucht am Ostrand der Endmoräne W von Eutin gefunden; beide Endmoränen sind ebenfalls jünger als die genannte Hauptmoräne M. Die Frage ist nur die, ob die Stauchwirkung in diesen Fällen direkt vom „lebenden “ Inlandeisrand oder indirekt durch Druck auf große schuttbedeckte Toteisfelder ausgeübt worden ist.

In Ostholstein ist eine zuverlässige Ermittlung der Randlage des Pommerschen Stadiums (wichtig für die relative Altersbestimmung der dortigen Interstadialbildungen) leider immer noch nicht gelungen. Der beste Kenner der Geologie der Umgebung von Lübeck, P. RANGE (1951) nimmt die Endmoräne dieses Stadiums zwischen Lübeck und Travemünde (im Raume Schwartau-Herrenfähre bei Lübeck) an; von dort verläuft sie nach P. Woldstedt (1950 S. 42) zunächst in nördlicher, dann in westlicher Richtung weiter.

Die erwähnten Bildungen entsprechen in jeder Hinsicht fossilführenden Ablagerungen, die die Hauptmasse des sogen. „Masurischen Interstadials“ darstellen. Sie sind also nicht auf Masuren beschränkt, hier aber auf dem sehr hügeligen Gelände am häufigsten, das in der Abschmelzphase des Pommerschen Stadiums ausgedehnte schuttbedeckte Toteisfelder trug, in Norddeutschland aber nicht nur auf dieses Abschmelzgebiet beschränkt, sondern gelegentlich auch im angrenzenden älteren Bereich der Weichselvereisung zu finden ist. Viele liegen zu Tage, andere sind am Rande oder ganz mit Grundmoräne oder Sand und Kies bedeckt. Das von B. KöRNKE entdeckte Inster-Interstadial im nördlichen Ostpreußen liegt, wie ich mich durch den Augenschein überzeugt habe, auf grauer Grundmoräne wie die "Masurischen Interstadialbildungen“ und meistens unter rotbrauner Grundmoräne, seltener unter Kamesbildungen und ist ganz zweifellos jünger als das Pommersche Stadium. Auch die zu Tage liegenden fossilführenden Ablagerungen des Daniglazials, die älter als die Zone Ia der zusammenhängenden Pollendiagramme sind, stammen aus der Zeit einer vorübergehenden Klimabesserung; am zweckmäßigsten könnte man sie als Daniglaziales (oder Pommersches) Interstadial zusammenfassend bezeichnen. Zwischen dem Frankfurter und dem Pommerschen Stadium, wie immer noch bisweilen das "Masurische Interstadial“ eingestuft wird, ist bisher noch nicht eine einzige interstadiale Ablagerung einwandfrei nachgewiesen, ebenso wenig zwischen dem Brandenburger und Frankfurter Stadium; im Bereich der Abschmelzphase des Brandenburger und Frankfurter Stadiums kam stellenweise noch spätglaziales Tieftauen von Toteis vor. Im Hochglazial konnte sicher die abkühlende Wirkung der Inlandeismasse etwaige vorübergehende Milderungen des Allgemeinklimas im Periglazial aufheben und so die Bildung fossilführender Ablagerungen verhindern; Interstadiale waren sicher nur vor und nach einem Hochglazial möglich.

5. Das Two Creeks Forest Bed-Interstadial in Nordamerika.

Die Richtigkeit der C 14-Datierung dieses Interstadials ist von E. Antevs zu Unrecht angezweifelt worden. Der Durchschnitt von 5 Altersbestimmungen von $\mathrm{Holz}$ aus diesem Interstadial durch W. F. LIBBY in Chicago (mit Verwendung von festem C) ergab $11404 \pm 350$ Jahre vor heute; die Altersbestimmung von Holz vom gleichen Fundort durch $\mathrm{H}$. E. Suess in Washington (mit Verwendung von $\mathrm{C}_{2} \mathrm{H}_{2}$ ) ergab als Durchschnitt von 2 Messungen $11410 \pm 180$ Jahre (Science Vol. 120, 1954, S. 472). 


\section{Schriftenverzeichnis}

Bertsch, K.: Die zeitliche Stellung der Renntierjäger an der Schussenquelle. - Schr. d. Ver. f. Geschichte d. Bodensees u. s. Umgebung 72. H., 19-30, 1953/54.

BRANDt, I.: Late-glacial macroscopic plant remains from Böllingsö. - Danmarks Geol. Unders. II. R. Nr. $80,156-158,1954$.

BRÜCKNER, G. H.: Artefakte der Jüngeren Hamburger Stufe in einer Grundmoräne bei Ostseebad Grömitz. - Meyniana (Kiel) 2, S. 191, 192, 1954.

De GeEr, E. H.: Conclusions from $\mathrm{C}^{14}$ and De GeER's chronology, Dani-Gotiglacial, with datings. - Geol. Fören. i Stockholm Förhandl. 73, 557-570, 1951. - - Skandinaviens geokronologi.-Ibid. 76, 299-329, 1954.

Frass, O.: Die neuesten Erfunde an der Schussenquelle bei Schussenried. - Jahresh. d. Ver. f. vaterländ. Naturk. in Württemberg 23, 48-74, 1867.

Gross, H.: Die Radiokarbon-Methode usw. - Eiszeitalter u. Gegenwart 2, 68-92, 1952. - Das Alleröd-Interstadial als Leithorizont usw. - Ibid. 4/5, 189-209, 1954.

Guenther, E. W.: Diluviale Säugetierfunde in Schleswig-Holstein und ihre zeitliche Einordnung. - Autorreferat eines Vortrags in Bad Segeberg am 21. 9. 1954.

Hallik, R., \& Grube, E.: Spät- und postglaziale Gyttja im Altmoränengebiet bei Elmshorn. N. Jb. f. Geol. u. Pal. Mh. 8, 315-322, 1954.

Iversen, Johs.: The late-glacial flora of Denmark and its relations to climate and soil. - Danmarks Geol. Unders. II. R. Nr. 80, 87-119, 1954.

LANG, H. D.: Ein Alleröd-Profil mit eingelagertem Laacher-See-Tuff bei Marburg/Lahn. - N. Jb. f. Geol. u. Pal. Mh., 362-372, 1954.

Lürzer, Eva von: Das Spätglazial im Egelseegebiet (Salzach-Vorlandgletscher). - Z. f. Gletscherk. u. Glazialgeol. 3, 83-90, 1954.

RANGE, P.: Neue spätquartäre pflanzenführende Ablagerungen in der Umgegend von Lübeck. Manuskript.

Rust, A.: Jungpaläolithische Artefakte in Würm-Grundmoränen Schleswig-Holsteins und ihr $\mathrm{C}^{14}$-Alter. - Autorreferat eines Vortrages auf der Jahresversammlung der Deutschen Quartärvereinigung in Bad Segeberg am 22. 9. 1954.

Sauvage, Jacqueline: Palynologie et pétrographie de tourbes et de sédiments de la Cuvette parisienne et des Ardennes. - Mém. Service de la carte géol. d'Alsace et de Lorraine Nr. 12, 71 S., 1954.

SснӥтrumpF, R.: Das Spätglazial. - Autorreferat eines Vortrags auf der Jahresvers. der Deutschen Quartärverein. in Bad Segeberg am 21. 9. 1954. - Dieses Jahrb. S. ...

SEIfERT, G.: Geschichte des Eisabbaus in Ostwagrien und Fehmarn. - Autorreferat eines Vortrags in Bad Segeberg (mit 3 Karten), 1954.

Manuskr. eingeg. 10. 2. 1955.

Anschrift des Verf.: Dr. Hugo Gross, Bamberg, Kunigundendamm 59. 